\title{
INFINITELY MANY SOLUTIONS FOR A DIRICHLET BOUNDARY VALUE PROBLEM DEPENDING ON TWO PARAMETERS
}

\author{
Ghasem A. Afrouzi and Armin Hadjian \\ University of Mazandaran, Iran
}

\begin{abstract}
In this paper, using Ricceri's variational principle, we prove the existence of infinitely many weak solutions for a Dirichlet doubly eigenvalue boundary value problem.
\end{abstract}

\section{INTRODUCTION}

The aim of this paper is to investigate the existence of infinitely many weak solutions for the following Dirichlet doubly eigenvalue boundary value problem on a bounded interval $[a, b]$ in $\mathbb{R}(a<b)$

(1.1) $\left\{\begin{array}{l}-u^{\prime \prime}+u h\left(x, u^{\prime}\right)=[\lambda f(x, u)+\mu g(x, u)+p(u)] h\left(x, u^{\prime}\right) \quad \text { in }(a, b), \\ u(a)=u(b)=0\end{array}\right.$

where $\lambda$ is a positive parameter, $\mu$ is a nonnegative parameter, $f, g:[a, b] \times$ $\mathbb{R} \rightarrow \mathbb{R}$ are $L^{1}$-Carathéodory functions, $p: \mathbb{R} \rightarrow \mathbb{R}$ is a Lipschitz continuous function with the Lipschitz constant $L>0$, i.e.,

$$
\left|p\left(t_{1}\right)-p\left(t_{2}\right)\right| \leq L\left|t_{1}-t_{2}\right|
$$

for every $t_{1}, t_{2} \in \mathbb{R}$, with $p(0)=0$, and $h:[a, b] \times \mathbb{R} \rightarrow[0,+\infty)$ is a bounded and continuous function with $m:=\inf _{(x, t) \in[a, b] \times \mathbb{R}} h(x, t)>0$.

Very recently a version of the infinitely many critical points theorem of Ricceri (see [20, Theorem 2.5]), the existence of an unbounded sequence of weak solutions for a Sturm-Liouville problem, having discontinuous nonlinearities, has been established in [4]. In a such approach, an appropriate

2010 Mathematics Subject Classification. 34B15, 35B38, 58E05.

Key words and phrases. Doubly eigenvalue boundary value problem, Ricceri's variational principle, infinitely many solutions. 
oscillating behavior of the nonlinear term either at infinity or at zero is required. This type of methodology has been used then in several works in order to obtain existence results for different kinds of problems (see $[1-3,5-13,15-17]$ and references therein).

In [1], the existence of infinitely many classical solutions for the following Dirichlet quasilinear system has been obtained

$$
\left\{\begin{array}{l}
-\left(p_{i}-1\right)\left|u_{i}^{\prime}(x)\right|^{p_{i}-2} u_{i}^{\prime \prime}(x)=\lambda F_{u_{i}}\left(x, u_{1}, \ldots, u_{n}\right) h_{i}\left(x, u_{i}^{\prime}\right), \quad x \in(a, b), \\
u_{i}(a)=u_{i}(b)=0, \quad \text { for } 1 \leq i \leq n,
\end{array}\right.
$$

where $p_{i}>1$ for $1 \leq i \leq n, \lambda$ is a positive parameter, $a, b \in \mathbb{R}$ with $a<b$, $h_{i}:[a, b] \times \mathbb{R} \rightarrow[0,+\infty)$ is a bounded and continuous function with $m_{i}:=$ $\inf _{(x, t) \in[a, b] \times \mathbb{R}} h_{i}(x, t)>0$ for $1 \leq i \leq n, F:[a, b] \times \mathbb{R}^{n} \rightarrow \mathbb{R}$ is a function such that the mapping $\left(t_{1}, t_{2}, \ldots, t_{n}\right) \rightarrow F\left(x, t_{1}, t_{2}, \ldots, t_{n}\right)$ is in $C^{1}$ in $\mathbb{R}^{n}$ for all $x \in[a, b], F_{u_{i}}$ is continuous in $[a, b] \times \mathbb{R}^{n}$ for $1 \leq i \leq n$, where $F_{u_{i}}$ denotes the partial derivative of $F$ with respect to $u_{i}$, and

$$
\sup _{\left|\left(t_{1}, \ldots, t_{n}\right)\right| \leq M}\left|F_{u_{i}}\left(\cdot, t_{1}, \ldots, t_{n}\right)\right| \in L^{1}([a, b])
$$

for all $M>0$ and all $1 \leq i \leq n$. Here, starting from the results obtained in [1] and with the same method, we are interested in looking for a class of perturbations, namely $\mu g+p$, for which (1.1) still preserves multiple solutions.

In particular, our goal in this paper is to obtain some sufficient conditions to guarantee that problem (1.1) has infinitely many weak solutions. To this end, we require that the primitive $F$ of $f$ satisfies a suitable oscillatory behavior either at infinity (for obtaining unbounded solutions) or at zero (for finding arbitrarily small solutions), while $G$, the primitive of $g$, has an appropriate growth (see Theorems 3.1 and 3.7). Our approach is fully variational and the main tool is a general critical point theorem contained in [4] (see Lemma 2.1 below and also [20]).

Here, as an example, we state a special case of our results.

THEOREM 1.1. Let $f: \mathbb{R} \rightarrow \mathbb{R}$ be a nonnegative continuous function and $p: \mathbb{R} \rightarrow \mathbb{R}$ a Lipschitz continuous function with the Lipschitz constant $1 \leq L<5$ and $p(0)=0$. Put $F(\xi):=\int_{0}^{\xi} f(t) d t$ for all $\xi \in \mathbb{R}$ and assume

$$
\liminf _{\xi \rightarrow+\infty} \frac{F(\xi)}{\xi^{2}}=0, \quad \limsup _{\xi \rightarrow+\infty} \frac{F(\xi)}{\xi^{2}}=+\infty .
$$

Then, the problem

$$
\left\{\begin{array}{l}
-u^{\prime \prime}+u=f(u)+p(u) \quad \text { in }(0,1), \\
u(0)=u(1)=0,
\end{array}\right.
$$

has a sequence of pairwise distinct classical solutions. 


\section{Preliminaries}

We shall prove our results applying the following smooth version of Theorem 2.1 of [4], which is a more precise version of Ricceri's Variational Principle [20, Theorem 2.5].

Lemma 2.1. Let $X$ be a reflexive real Banach space, let $\Phi, \Psi: X \rightarrow \mathbb{R}$ be two Gâteaux differentiable functionals such that $\Phi$ is sequentially weakly lower semicontinuous, strongly continuous and coercive, and $\Psi$ is sequentially weakly upper semicontinuous. For every $r>\inf _{X} \Phi$, let

$$
\begin{gathered}
\varphi(r):=\inf _{u \in \Phi^{-1}(-\infty, r)} \frac{\left(\sup _{v \in \Phi^{-1}(-\infty, r)} \Psi(v)\right)-\Psi(u)}{r-\Phi(u)}, \\
\gamma:=\liminf _{r \rightarrow+\infty} \varphi(r), \quad \text { and } \quad \delta:=\liminf _{r \rightarrow\left(\inf _{X} \Phi\right)^{+}} \varphi(r) .
\end{gathered}
$$

Then:

(a) For every $r>\inf _{X} \Phi$ and every $\lambda \in(0,1 / \varphi(r))$, the restriction of the functional

$$
I_{\lambda}:=\Phi-\lambda \Psi
$$

to $\Phi^{-1}(-\infty, r)$ admits a global minimum, which is a critical point (local minimum) of $I_{\lambda}$ in $X$.

(b) If $\gamma<+\infty$, then for each $\lambda \in(0,1 / \gamma)$, the following alternative holds: either

$\left(\mathrm{b}_{1}\right) I_{\lambda}$ possesses a global minimum, or

$\left(\mathrm{b}_{2}\right)$ there is a sequence $\left\{u_{n}\right\}$ of critical points (local minima) of $I_{\lambda}$ such that

$$
\lim _{n \rightarrow+\infty} \Phi\left(u_{n}\right)=+\infty .
$$

(c) If $\delta<+\infty$, then for each $\lambda \in(0,1 / \delta)$, the following alternative holds: either

$\left(c_{1}\right)$ there is a global minimum of $\Phi$ which is a local minimum of $I_{\lambda}$, or

$\left(\mathrm{c}_{2}\right)$ there is a sequence $\left\{u_{n}\right\}$ of pairwise distinct critical points (local minima) of $I_{\lambda}$ that converges weakly to a global minimum of $\Phi$.

Let $f, g:[a, b] \times \mathbb{R} \rightarrow \mathbb{R}$ be two $L^{1}$-Carathéodory functions, $p: \mathbb{R} \rightarrow \mathbb{R}$ be a Lipschitz continuous function with the Lipschitz constant $L>0$, i.e.,

$$
\left|p\left(t_{1}\right)-p\left(t_{2}\right)\right| \leq L\left|t_{1}-t_{2}\right|
$$

for every $t_{1}, t_{2} \in \mathbb{R}$, with $p(0)=0$, and $h:[a, b] \times \mathbb{R} \rightarrow[0,+\infty)$ be a bounded and continuous function with $m:=\inf _{(x, t) \in[a, b] \times \mathbb{R}} h(x, t)>0$.

We recall that $f:[a, b] \times \mathbb{R} \rightarrow \mathbb{R}$ is an $L^{1}$-Carathéodory function if

(a) $x \mapsto f(x, \xi)$ is measurable for every $\xi \in \mathbb{R}$;

(b) $\xi \mapsto f(x, \xi)$ is continuous for almost every $x \in[a, b]$; 
(c) for every $\rho>0$ there is a function $l_{\rho} \in L^{1}([a, b])$ such that

$$
\sup _{|\xi| \leq \rho}|f(x, \xi)| \leq l_{\rho}(x)
$$

for almost every $x \in[a, b]$.

Corresponding to $f, g, p$ and $h$ we introduce the functions $F, G:[a, b] \times$ $\mathbb{R} \rightarrow \mathbb{R}, P: \mathbb{R} \rightarrow \mathbb{R}$ and $H:[a, b] \times \mathbb{R} \rightarrow[0,+\infty)$, respectively, as follows

$$
\begin{gathered}
F(x, t):=\int_{0}^{t} f(x, \xi) d \xi, \quad G(x, t):=\int_{0}^{t} g(x, \xi) d \xi, \\
P(t):=-\int_{0}^{t} p(\xi) d \xi
\end{gathered}
$$

and

$$
H(x, t):=\int_{0}^{t}\left(\int_{0}^{\tau} \frac{1}{h(x, \delta)} d \delta\right) d \tau,
$$

for all $x \in[a, b]$ and $t \in \mathbb{R}$.

Here and in the following, we let $X$ be the Sobolev space $W_{0}^{1,2}([a, b])$ equipped with the norm

$$
\|u\|:=\left(\int_{a}^{b}\left|u^{\prime}(x)\right|^{2} d x\right)^{1 / 2} .
$$

We say that a function $u \in X$ is a weak solution of problem (1.1) if

$$
\begin{aligned}
& \int_{a}^{b}\left(\int_{0}^{u^{\prime}(x)} \frac{1}{h(x, \tau)} d \tau\right) v^{\prime}(x) d x+\int_{a}^{b} u(x) v(x) d x \\
& -\lambda \int_{a}^{b} f(x, u(x)) v(x) d x-\mu \int_{a}^{b} g(x, u(x)) v(x) d x-\int_{a}^{b} p(u(x)) v(x) d x=0
\end{aligned}
$$

for all $v \in X$.

By standard regularity results, if $f$ and $g$ are continuous functions, then weak solutions of problem (1.1) belong to $C^{2}([a, b])$, thus they are classical solutions.

In the following, let $M:=\sup _{(x, t) \in[a, b] \times \mathbb{R}} h(x, t)$ and suppose that the Lipschitz constant $L>0$ of the function $p$ satisfies the following condition:

$\left(\mathrm{A}_{0}\right) L \geq 1$ and $M(L-1)(b-a)^{2}<4$.

Now, put

$$
\begin{aligned}
k_{1} & :=\frac{4+m(1+L)(b-a)^{2}}{8 m}, \\
k_{2} & :=\frac{4+M(1-L)(b-a)^{2}}{2 M}, \\
A & :=\liminf _{\xi \rightarrow+\infty} \frac{\int_{a}^{b} \sup _{|t| \leq \xi} F(x, t) d x}{\xi^{2}},
\end{aligned}
$$


and

$$
B:=\limsup _{\xi \rightarrow+\infty} \frac{\int_{a+\alpha}^{b-\beta} F(x, \xi) d x}{\xi^{2}} .
$$

Here, $\alpha$ and $\beta$ are two positive constants, such that $\alpha+\beta<b-a$.

For other basic notations and definitions, we refer the reader to $[14,19,22]$.

\section{MAIN RESUlts}

We formulate our main result as follows.

TheOREM 3.1. Assume that there exist two positive constants $\alpha$ and $\beta$ with $\alpha+\beta<b-a$ such that

$\left(\mathrm{A}_{1}\right) F(x, t) \geq 0$ for each $(x, t) \in([a, a+\alpha] \cup[b-\beta, b]) \times \mathbb{R}$;

$\left(\mathrm{A}_{2}\right) A<\left(\frac{\alpha \beta}{\alpha+\beta} \frac{k_{2}}{(b-a) k_{1}}\right) B$.

Then, by setting

$$
\lambda_{1}:=\frac{(\alpha+\beta) k_{1}}{\alpha \beta B}, \quad \lambda_{2}:=\frac{k_{2}}{(b-a) A},
$$

for every $\lambda \in\left(\lambda_{1}, \lambda_{2}\right)$ and for every arbitrary $L^{1}$-Carathéodory function $g$ : $[a, b] \times \mathbb{R} \rightarrow \mathbb{R}$, whose potential $G$ is a nonnegative function satisfying the condition

$$
g_{\infty}:=\lim _{\xi \rightarrow+\infty} \frac{\int_{a}^{b} \sup _{|t| \leq \xi} G(x, t) d x}{\xi^{2}}<+\infty,
$$

if we put

$$
\mu_{g, \lambda}:=\frac{k_{2}}{(b-a) g_{\infty}}\left(1-\lambda \frac{(b-a) A}{k_{2}}\right),
$$

where $\mu_{g, \lambda}=+\infty$ when $g_{\infty}=0$, for every $\mu \in\left[0, \mu_{g, \lambda}\right)$ problem (1.1) has an unbounded sequence of weak solutions in $X$.

Proof. Our aim is to apply Lemma 2.1(b) to our problem. To this end, fix $\bar{\lambda} \in\left(\lambda_{1}, \lambda_{2}\right)$ and let $g$ be a function that satisfies the condition (3.1). Since $\bar{\lambda}<\lambda_{2}$, we have

$$
\mu_{g, \bar{\lambda}}=\frac{k_{2}}{(b-a) g_{\infty}}\left(1-\bar{\lambda} \frac{(b-a) A}{k_{2}}\right)>0 .
$$

Now fix $\bar{\mu} \in\left(0, \mu_{g, \bar{\lambda}}\right)$ and set

$$
J(x, \xi):=F(x, \xi)+\frac{\bar{\mu}}{\bar{\lambda}} G(x, \xi)
$$

for all $(x, \xi) \in[a, b] \times \mathbb{R}$. For each $u \in X$, we let the functionals $\Phi, \Psi: X \rightarrow \mathbb{R}$ be defined by

$$
\Phi(u):=\int_{a}^{b} H\left(x, u^{\prime}(x)\right) d x+\frac{1}{2} \int_{a}^{b}|u(x)|^{2} d x+\int_{a}^{b} P(u(x)) d x
$$


and

$$
\Psi(u):=\int_{a}^{b} J(x, u(x)) d x
$$

and put

$$
I_{\bar{\lambda}}(u):=\Phi(u)-\bar{\lambda} \Psi(u), \quad u \in X
$$

Note that the weak solutions of (1.1) are exactly the critical points of $I_{\bar{\lambda}}$. The functionals $\Phi$ and $\Psi$ satisfy the regularity assumptions of Lemma 2.1. Indeed, by standard arguments, we have that $\Phi$ is Gâteaux differentiable and sequentially weakly lower semicontinuous and its Gâteaux derivative is the functional $\Phi^{\prime}(u) \in X^{*}$, given by

$$
\begin{aligned}
\Phi^{\prime}(u)(v)= & \int_{a}^{b}\left(\int_{0}^{u^{\prime}(x)} \frac{1}{h(x, \tau)} d \tau\right) v^{\prime}(x) d x \\
& +\int_{a}^{b} u(x) v(x) d x-\int_{a}^{b} p(u(x)) v(x) d x
\end{aligned}
$$

for every $v \in X$. Furthermore, the differential $\Phi^{\prime}: X \rightarrow X^{*}$ is a Lipschitzian operator. Indeed, for any $u, v \in X$, there holds

$$
\begin{aligned}
\left\|\Phi^{\prime}(u)-\Phi^{\prime}(v)\right\|_{X^{*}} & =\sup _{\|w\| \leq 1}\left|\left(\Phi^{\prime}(u)-\Phi^{\prime}(v), w\right)\right| \\
\leq & \sup _{\|w\| \leq 1} \int_{a}^{b}\left|\int_{u^{\prime}(x)}^{v^{\prime}(x)} \frac{1}{h(x, \tau)} d \tau\right|\left|w^{\prime}(x)\right| d x \\
& +\sup _{\|w\| \leq 1} \int_{a}^{b}|u(x)-v(x)||w(x)| d x \\
& +\sup _{\|w\| \leq 1} \int_{a}^{b}|p(u(x))-p(v(x))||w(x)| d x \\
\leq & \frac{1}{m}\|u-v\|+(1+L) \sup _{\|w\| \leq 1}\|u-v\|_{L^{2}([a, b])}\|w\|_{L^{2}([a, b])} .
\end{aligned}
$$

Recalling that $p$ is Lipschitz continuous, $h$ is bounded away from zero, and the embedding $X \hookrightarrow L^{2}([a, b])$ is compact, the claim is true. In particular, we derive that $\Phi$ is continuously differentiable. The inequality

$$
\max _{x \in[a, b]}|u(x)| \leq \frac{(b-a)^{1 / 2}}{2}\|u\|, \quad \text { for all } u \in X
$$


([21]) yields for any $u, v \in X$ the estimate

$$
\begin{aligned}
\left(\Phi^{\prime}(u)-\Phi^{\prime}(v), u-v\right)= & \int_{a}^{b}\left(\int_{v^{\prime}(x)}^{u^{\prime}(x)} \frac{1}{h(x, \tau)} d \tau\right)\left(u^{\prime}(x)-v^{\prime}(x)\right) d x \\
& +\int_{a}^{b}|u(x)-v(x)|^{2} d x \\
& -\int_{a}^{b}(p(u(x))-p(v(x)))(u(x)-v(x)) d x \\
\geq & \frac{1}{M}\|u-v\|^{2}+(1-L)\|u-v\|_{L^{2}([a, b])}^{2} \\
\geq & \frac{k_{2}}{2}\|u-v\|^{2} .
\end{aligned}
$$

By the assumption $\left(\mathrm{A}_{0}\right)$, it turns out that $\Phi^{\prime}$ is a strongly monotone operator. So, by applying Minty-Browder theorem ([22, Theorem 26.A]), $\Phi^{\prime}: X \rightarrow X^{*}$ admits a Lipschitz continuous inverse. On the other hand, the fact that $X$ is compactly embedded into $C^{0}([a, b])$ implies that the functional $\Psi$ is well defined, continuously Gâteaux differentiable and with compact derivative, whose Gâteaux derivative is given by

$$
\Psi^{\prime}(u)(v)=\int_{a}^{b} f(x, u(x)) v(x) d x+\frac{\bar{\mu}}{\bar{\lambda}} \int_{a}^{b} g(x, u(x)) v(x) d x
$$

for every $v \in X$. Hence $\Psi$ is sequentially weakly (upper) continuous (see [22, Corollary 41.9]).

Since $p$ is Lipschitz continuous and satisfies $p(0)=0$, while $h$ is bounded away from zero, we have from (3.2) that

$$
\Phi(u) \geq \frac{k_{2}}{4}\|u\|^{2},
$$

for all $u \in X$, and so $\Phi$ is coercive.

First of all, we will show that $\bar{\lambda}<1 / \gamma$. Hence, let $\left\{\xi_{n}\right\}$ be a sequence of positive numbers such that $\lim _{n \rightarrow+\infty} \xi_{n}=+\infty$ and

$$
\lim _{n \rightarrow+\infty} \frac{\int_{a}^{b} \sup _{|t| \leq \xi_{n}} F(x, t) d x}{\xi_{n}^{2}}=A .
$$

Put

$$
r_{n}:=\frac{k_{2}}{b-a} \xi_{n}^{2}
$$


for all $n \in \mathbb{N}$. Then, for all $v \in X$ with $\Phi(v)<r_{n}$, together with (3.2) and (3.3), we have $\|v\|_{\infty}<\xi_{n}$. Note that $\Phi(0)=\Psi(0)=0$. Then, for all $n \in \mathbb{N}$,

$$
\begin{aligned}
\varphi\left(r_{n}\right) & =\inf _{u \in \Phi^{-1}\left(-\infty, r_{n}\right)} \frac{\left(\sup _{v \in \Phi^{-1}\left(-\infty, r_{n}\right)} \Psi(v)\right)-\Psi(u)}{r_{n}-\Phi(u)} \\
& \leq \frac{\sup _{v \in \Phi^{-1}\left(-\infty, r_{n}\right)} \Psi(v)}{r_{n}} \\
& \leq \frac{b-a}{k_{2}} \frac{\int_{a}^{b} \sup _{|t| \leq \xi_{n}} J(x, t) d x}{\xi_{n}^{2}} \\
& =\frac{b-a}{k_{2}} \frac{\int_{a}^{b} \sup _{|t| \leq \xi_{n}}\left[F(x, t)+\frac{\bar{\mu}}{\bar{\lambda}} G(x, t)\right] d x}{\xi_{n}^{2}} \\
& \leq \frac{b-a}{k_{2}}\left[\frac{\int_{a}^{b} \sup _{|t| \leq \xi_{n}} F(x, t) d x}{\xi_{n}^{2}}+\frac{\bar{\mu}}{\bar{\lambda}} \frac{\int_{a}^{b} \sup _{|t| \leq \xi_{n}} G(x, t) d x}{\xi_{n}^{2}}\right] .
\end{aligned}
$$

Moreover, from the assumption $\left(\mathrm{A}_{2}\right)$ and the condition (3.1) we have $A<+\infty$ and

$$
\lim _{n \rightarrow+\infty} \frac{\int_{a}^{b} \sup _{|t| \leq \xi_{n}} G(x, t) d x}{\xi_{n}^{2}}=g_{\infty}
$$

Therefore,

$$
\gamma \leq \liminf _{n \rightarrow+\infty} \varphi\left(r_{n}\right) \leq \frac{b-a}{k_{2}}\left(A+\frac{\bar{\mu}}{\bar{\lambda}} g_{\infty}\right)<+\infty
$$

The assumption $\bar{\mu} \in\left(0, \mu_{g, \bar{\lambda}}\right)$ immediately yields

$$
\gamma \leq \frac{b-a}{k_{2}}\left(A+\frac{\bar{\mu}}{\bar{\lambda}} g_{\infty}\right)<\frac{b-a}{k_{2}} A+\frac{1-\frac{b-a}{k_{2}} \bar{\lambda} A}{\bar{\lambda}} .
$$

Hence,

$$
\bar{\lambda}=\frac{1}{\frac{b-a}{k_{2}} A+\left(1-\frac{b-a}{k_{2}} \bar{\lambda} A\right) / \bar{\lambda}}<\frac{1}{\gamma} .
$$

Let $\bar{\lambda}$ be fixed. We claim that the functional $I_{\bar{\lambda}}$ is unbounded from below. Since

$$
\frac{1}{\bar{\lambda}}<\frac{\alpha \beta B}{(\alpha+\beta) k_{1}},
$$

there exists a sequence $\left\{\eta_{n}\right\}$ of positive numbers and $\tau>0$ such that $\lim _{n \rightarrow+\infty} \eta_{n}=+\infty$ and

$$
\frac{1}{\bar{\lambda}}<\tau<\frac{\alpha \beta}{(\alpha+\beta) k_{1}} \frac{\int_{a+\alpha}^{b-\beta} F\left(x, \eta_{n}\right) d x}{\eta_{n}^{2}}
$$


for each $n \in \mathbb{N}$ large enough. For all $n \in \mathbb{N}$ define

$$
w_{n}(x):= \begin{cases}\frac{\eta_{n}}{\alpha}(x-a), & \text { if } a \leq x<a+\alpha, \\ \eta_{n}, & \text { if } a+\alpha \leq x \leq b-\beta, \\ \frac{\eta_{n}}{\beta}(b-x), & \text { if } b-\beta<x \leq b .\end{cases}
$$

For any fixed $n \in \mathbb{N}$, it is easy to see that $w_{n} \in X$ and, in particular, one has

$$
\left\|w_{n}\right\|^{2}=\frac{\alpha+\beta}{\alpha \beta} \eta_{n}^{2}
$$

and so

$$
\Phi\left(w_{n}\right) \leq k_{1}\left\|w_{n}\right\|^{2}=\frac{(\alpha+\beta) k_{1}}{\alpha \beta} \eta_{n}^{2} .
$$

On the other hand, bearing $\left(\mathrm{A}_{1}\right)$ in mind and since $G$ is nonnegative, from the definition of $\Psi$, we infer

$$
\Psi\left(w_{n}\right)=\int_{a}^{b}\left[F\left(x, w_{n}(x)\right)+\frac{\bar{\mu}}{\bar{\lambda}} G\left(x, w_{n}(x)\right)\right] d x \geq \int_{a+\alpha}^{b-\beta} F\left(x, \eta_{n}\right) d x .
$$

By (3.6), (3.8) and (3.9), we see that

$$
I_{\bar{\lambda}}\left(w_{n}\right) \leq \frac{(\alpha+\beta) k_{1} \eta_{n}^{2}}{\alpha \beta}-\bar{\lambda} \int_{a+\alpha}^{b-\beta} F\left(x, \eta_{n}\right) d x<\frac{(\alpha+\beta) k_{1} \eta_{n}^{2}}{\alpha \beta}(1-\bar{\lambda} \tau)
$$

for every $n \in \mathbb{N}$ large enough. Since $\bar{\lambda} \tau>1$ and $\eta_{n} \rightarrow+\infty$ as $n \rightarrow+\infty$, we have

$$
\lim _{n \rightarrow+\infty} I_{\bar{\lambda}}\left(w_{n}\right)=-\infty
$$

Then, the functional $I_{\bar{\lambda}}$ is unbounded from below, and it follows that $I_{\bar{\lambda}}$ has no global minimum. Therefore, by Lemma 2.1(b), there exists a sequence $\left\{u_{n}\right\}$ of critical points of $I_{\bar{\lambda}}$ such that

$$
\lim _{n \rightarrow+\infty}\left\|u_{n}\right\|=+\infty
$$

and the conclusion is achieved.

Remark 3.2. Under the conditions $A=0$ and $B=+\infty$, from Theorem 3.1 we see that for every $\lambda>0$ and for each $\mu \in\left[0, \frac{k_{2}}{(b-a) g_{\infty}}\right)$, problem (1.1) admits a sequence of weak solutions which is unbounded in $X$. Moreover, if $g_{\infty}=0$, the result holds for every $\lambda>0$ and $\mu \geq 0$.

The following result is a special case of Theorem 3.1 with $\mu=0$.

Theorem 3.3. Assume that all the assumptions in Theorem 3.1 hold. Then, for each

$$
\lambda \in\left(\frac{(\alpha+\beta) k_{1}}{\alpha \beta B}, \frac{k_{2}}{(b-a) A}\right),
$$


the problem

$$
\left\{\begin{array}{l}
-u^{\prime \prime}+u h\left(x, u^{\prime}\right)=[\lambda f(x, u)+p(u)] h\left(x, u^{\prime}\right) \quad \text { in }(a, b), \\
u(a)=u(b)=0,
\end{array}\right.
$$

has an unbounded sequence of weak solutions in $X$.

REMARK 3.4. Theorem 1.1 in the Introduction immediately follows from Theorem 3.3, setting $h(x, t) \equiv 1$ for all $(x, t) \in[0,1] \times \mathbb{R}$.

Here we point out the following consequences of Theorem 3.3.

Corollary 3.5. Assume that there exist two positive constants $\alpha$ and $\beta$ with $\alpha+\beta<b-a$ such that the assumption $\left(\mathrm{A}_{1}\right)$ in Theorem 3.1 holds. Suppose that

Then, the problem

$$
A<\frac{k_{2}}{b-a}, \quad B>\frac{(\alpha+\beta) k_{1}}{\alpha \beta} .
$$

$$
\left\{\begin{array}{l}
-u^{\prime \prime}+u h\left(x, u^{\prime}\right)=[f(x, u)+p(u)] h\left(x, u^{\prime}\right) \quad \text { in }(a, b), \\
u(a)=u(b)=0,
\end{array}\right.
$$

has an unbounded sequence of weak solutions in $X$.

Corollary 3.6. Let $g_{1}:[a, b] \rightarrow \mathbb{R}$ be a nonnegative continuous function, and put $G_{1}(t):=\int_{0}^{t} g_{1}(\xi) d \xi$ for all $t \in \mathbb{R}$. Assume that

$\left(\mathrm{A}_{3}\right) \liminf _{\xi \rightarrow+\infty} \frac{G_{1}(\xi)}{\xi^{2}}<+\infty$;

$\left(\mathrm{A}_{4}\right) \limsup _{\xi \rightarrow+\infty} \frac{G_{1}(\xi)}{\xi^{2}}=+\infty$.

Then, for every $\alpha_{i} \in L^{1}([a, b])$ for $1 \leq i \leq n$, with $\min _{x \in[a, b]}\left\{\alpha_{i}(x): 1 \leq i \leq\right.$ $n\} \geq 0$ and with $\alpha_{1} \neq 0$, and for every nonnegative continuous $g_{i}: \mathbb{R} \rightarrow \mathbb{R}$ for $2 \leq i \leq n$ satisfying

$$
\max \left\{\sup _{\xi \in \mathbb{R}} G_{i}(\xi): 2 \leq i \leq n\right\} \leq 0
$$

and

$$
\min \left\{\liminf _{\xi \rightarrow+\infty} \frac{G_{i}(\xi)}{\xi^{2}}: 2 \leq i \leq n\right\}>-\infty,
$$

where $G_{i}(t):=\int_{0}^{t} g_{i}(\xi) d \xi$ for all $t \in \mathbb{R}$ for $2 \leq i \leq n$, for each

$$
\lambda \in\left(0, \frac{\frac{k_{2}}{b-a}}{\left(\int_{a}^{b} \alpha_{1}(x) d x\right) \liminf _{\xi \rightarrow+\infty} \frac{G_{1}(\xi)}{\xi^{2}}}\right),
$$

the problem

$$
\left\{\begin{array}{l}
-u^{\prime \prime}+u h\left(x, u^{\prime}\right)=\left[\lambda \sum_{i=1}^{n} \alpha_{i}(x) g_{i}(u)+p(u)\right] h\left(x, u^{\prime}\right) \quad \text { in }(a, b), \\
u(a)=u(b)=0,
\end{array}\right.
$$

has an unbounded sequence of weak solutions in $X$. 
Proof. Put $f(x, t)=\sum_{i=1}^{n} \alpha_{i}(x) g_{i}(t)$ for all $(x, t) \in[a, b] \times \mathbb{R}$. The assumption $\left(\mathrm{A}_{4}\right)$ in conjunction with the condition

$$
\min \left\{\liminf _{\xi \rightarrow+\infty} \frac{G_{i}(\xi)}{\xi^{2}}: 2 \leq i \leq n\right\}>-\infty
$$

yields

$$
\limsup _{\xi \rightarrow+\infty} \frac{\int_{a+\alpha}^{b-\beta} F(x, \xi) d x}{\xi^{2}}=\limsup _{\xi \rightarrow+\infty} \frac{\sum_{i=1}^{n}\left(G_{i}(\xi) \int_{a+\alpha}^{b-\beta} \alpha_{i}(x) d x\right)}{\xi^{2}}=+\infty .
$$

Moreover, the assumption $\left(\mathrm{A}_{3}\right)$ along with the condition

ensures

$$
\max \left\{\sup _{\xi \in \mathbb{R}} G_{i}(\xi): 2 \leq i \leq n\right\} \leq 0
$$

$$
\liminf _{\xi \rightarrow+\infty} \frac{\int_{a}^{b} \sup _{|t| \leq \xi} F(x, t) d x}{\xi^{2}} \leq\left(\int_{a}^{b} \alpha_{1}(x) d x\right) \liminf _{\xi \rightarrow+\infty} \frac{G_{1}(\xi)}{\xi^{2}}<+\infty .
$$

Hence, the conclusion follows from Theorem 3.3.

Put

$$
A^{\prime}:=\liminf _{\xi \rightarrow 0^{+}} \frac{\int_{a}^{b} \sup _{|t| \leq \xi} F(x, t) d x}{\xi^{2}}
$$

and

$$
B^{\prime}:=\limsup _{\xi \rightarrow 0^{+}} \frac{\int_{a+\alpha}^{b-\beta} F(x, \xi) d x}{\xi^{2}} .
$$

Using Lemma 2.1(c) and arguing as in the proof of Theorem 3.1, we can obtain the following result.

THEOREM 3.7. Assume that there exist two positive constants $\alpha$ and $\beta$ with $\alpha+\beta<b-a$ such that $\left(\mathrm{A}_{1}\right)$ in Theorem 3.1 holds and

$\left(\mathrm{A}_{5}\right) A^{\prime}<\left(\frac{\alpha \beta}{\alpha+\beta} \frac{k_{2}}{(b-a) k_{1}}\right) B^{\prime}$.

Then, setting

$$
\lambda_{3}:=\frac{(\alpha+\beta) k_{1}}{\alpha \beta B^{\prime}}, \quad \lambda_{4}:=\frac{k_{2}}{(b-a) A^{\prime}},
$$

for every $\lambda \in\left(\lambda_{3}, \lambda_{4}\right)$ and for every arbitrary $L^{1}$-Carathéodory function $g$ : $[a, b] \times \mathbb{R} \rightarrow \mathbb{R}$, whose potential $G$ is a nonnegative function satisfying the condition

$$
g_{0}:=\lim _{\xi \rightarrow 0^{+}} \frac{\int_{a}^{b} \sup _{|t| \leq \xi} G(x, t) d x}{\xi^{2}}<+\infty
$$

if we put

$$
\mu_{g, \lambda}^{\prime}:=\frac{k_{2}}{(b-a) g_{0}}\left(1-\lambda \frac{(b-a) A^{\prime}}{k_{2}}\right)
$$


where $\mu_{g, \lambda}^{\prime}=+\infty$ when $g_{0}=0$, for every $\mu \in\left[0, \mu_{g, \lambda}^{\prime}\right)$ problem (1.1) has a sequence of weak solutions, which strongly converges to 0 in $X$.

Proof. Fix $\bar{\lambda} \in\left(\lambda_{3}, \lambda_{4}\right)$ and let $g$ be a function that satisfies the condition (3.11). Since $\bar{\lambda}<\lambda_{4}$, one has

$$
\mu_{g, \bar{\lambda}}^{\prime}=\frac{k_{2}}{(b-a) g_{0}}\left(1-\bar{\lambda} \frac{(b-a) A^{\prime}}{k_{2}}\right)>0 .
$$

Now fix $\bar{\mu} \in\left(0, \mu_{g, \bar{\lambda}}^{\prime}\right)$ and set

$$
J(x, \xi):=F(x, \xi)+\frac{\bar{\mu}}{\bar{\lambda}} G(x, \xi)
$$

for all $(x, \xi) \in[a, b] \times \mathbb{R}$. We take $\Phi, \Psi$ and $I_{\bar{\lambda}}$ as in the proof of Theorem 3.1. Now, as has been pointed out before, the functionals $\Phi$ and $\Psi$ satisfy the regularity assumptions required in Lemma 2.1. As first step, we will prove that $\bar{\lambda}<1 / \delta$. Then, let $\left\{\xi_{n}\right\}$ be a sequence of positive numbers such that $\lim _{n \rightarrow+\infty} \xi_{n}=0$ and

$$
\lim _{n \rightarrow+\infty} \frac{\int_{a}^{b} \sup _{|t| \leq \xi_{n}} F(x, t) d x}{\xi_{n}^{2}}=A^{\prime} .
$$

By the fact that $\inf _{X} \Phi=0$ and the definition of $\delta$, we have $\delta=$ $\liminf _{r \rightarrow 0^{+}} \varphi(r)$. Then, as in showing (3.5) in the proof of Theorem 3.1, we can prove that $\delta<+\infty$.

From $\bar{\mu} \in\left(0, \mu_{g, \bar{\lambda}}^{\prime}\right)$, the following inequalities hold

$$
\delta \leq \frac{b-a}{k_{2}}\left(A^{\prime}+\frac{\bar{\mu}}{\bar{\lambda}} g_{0}\right)<\frac{b-a}{k_{2}} A^{\prime}+\frac{1-\frac{b-a}{k_{2}} \bar{\lambda} A^{\prime}}{\bar{\lambda}} .
$$

Hence,

$$
\bar{\lambda}=\frac{1}{\frac{b-a}{k_{2}} A^{\prime}+\left(1-\frac{b-a}{k_{2}} \bar{\lambda} A^{\prime}\right) / \bar{\lambda}}<\frac{1}{\delta} .
$$

Let $\bar{\lambda}$ be fixed. We claim that the functional $I_{\bar{\lambda}}$ has not a local minimum at zero. Since

$$
\frac{1}{\bar{\lambda}}<\frac{\alpha \beta B^{\prime}}{(\alpha+\beta) k_{1}}
$$

there exists a sequence $\left\{\eta_{n}\right\}$ of positive numbers and $\tau>0$ such that $\lim _{n \rightarrow+\infty} \eta_{n}=0$ and

$$
\frac{1}{\bar{\lambda}}<\tau<\frac{\alpha \beta}{(\alpha+\beta) k_{1}} \frac{\int_{a+\alpha}^{b-\beta} F\left(x, \eta_{n}\right) d x}{\eta_{n}^{2}}
$$


for each $n \in \mathbb{N}$ large enough. For all $n \in \mathbb{N}$ define $w_{n}(x)$ as in (3.7) with the above $\eta_{n}$. Note that $\bar{\lambda} \tau>1$. Then, as in showing (3.10), we can obtain that

$$
\begin{aligned}
I_{\bar{\lambda}}\left(w_{n}\right) & =\Phi\left(w_{n}\right)-\bar{\lambda} \Psi\left(w_{n}\right) \\
& \leq \frac{(\alpha+\beta) k_{1} \eta_{n}^{2}}{\alpha \beta}-\bar{\lambda} \int_{a+\alpha}^{b-\beta} F\left(x, \eta_{n}\right) d x \\
& <\frac{(\alpha+\beta) k_{1} \eta_{n}^{2}}{\alpha \beta}(1-\bar{\lambda} \tau)<0
\end{aligned}
$$

for every $n \in \mathbb{N}$ large enough. Then, since

$$
\lim _{n \rightarrow+\infty} I_{\bar{\lambda}}\left(w_{n}\right)=I_{\bar{\lambda}}(0)=0,
$$

we see that 0 is not a local minimum of $I_{\bar{\lambda}}$. This, together with the fact that 0 is the only global minimum of $\Phi$, shows that the functional $I_{\bar{\lambda}}$ has not a local minimum at zero. Therefore, by Lemma $2.1(\mathrm{c})$, there exists a sequence $\left\{u_{n}\right\}$ of critical points of $I_{\bar{\lambda}}$ which converges weakly to 0 . In view of the fact that the embedding $X \hookrightarrow C^{0}([a, b])$ is compact, we know that the critical points converge strongly to zero, and the proof is complete.

Remark 3.8. Under the conditions $A^{\prime}=0$ and $B^{\prime}=+\infty$, Theorem 3.7 ensures that for every $\lambda>0$ and for each $\mu \in\left[0, \frac{k_{2}}{(b-a) g_{0}}\right)$, problem (1.1) admits a sequence of weak solutions, which strongly converges to 0 in $X$. Moreover, if $g_{0}=0$, the result holds for every $\lambda>0$ and $\mu \geq 0$.

Remark 3.9. Applying Theorem 3.7, results similar to Theorems 1.1 and 3.3, and Corollaries 3.5 and 3.6 can be obtained. We omit the discussions here.

We conclude this paper with the following example to illustrate our results.

Example 3.10. Put

$$
a_{n}:=\frac{2 n !(n+2) !-1}{4(n+1) !}, \quad b_{n}:=\frac{2 n !(n+2) !+1}{4(n+1) !} .
$$

for every $n \in \mathbb{N}$, and define the nonnegative continuous function $f: \mathbb{R} \rightarrow \mathbb{R}$ by

$f(\xi)= \begin{cases}\frac{32(n+1) !^{2}\left[(n+1) !^{2}-n !^{2}\right]}{\pi} \sqrt{\frac{1}{16(n+1) !^{2}}-\left(\xi-\frac{n !(n+2)}{2}\right)^{2}}, & \text { if } \xi \in \bigcup_{n \in \mathbb{N}}\left[a_{n}, b_{n}\right], \\ 0, & \text { otherwise. }\end{cases}$

One has

$$
\int_{n !}^{(n+1) !} f(t) d t=\int_{a_{n}}^{b_{n}} f(t) d t=(n+1) !^{2}-n !^{2}
$$

for every $n \in \mathbb{N}$. Then, one has

$$
\lim _{n \rightarrow+\infty} \frac{F\left(a_{n}\right)}{a_{n}^{2}}=0 \quad \text { and } \quad \lim _{n \rightarrow+\infty} \frac{F\left(b_{n}\right)}{b_{n}^{2}}=4 .
$$


Note that there is no sequence $\left\{c_{n}\right\}$ such that $\lim _{n \rightarrow+\infty} c_{n}=+\infty$ and $\lim _{n \rightarrow+\infty} F\left(c_{n}\right) / c_{n}^{2}>4$. Therefore,

$$
\liminf _{\xi \rightarrow+\infty} \frac{F(\xi)}{\xi^{2}}=0 \quad \text { and } \quad \limsup _{\xi \rightarrow+\infty} \frac{F(\xi)}{\xi^{2}}=4 .
$$

Hence, by choosing $[a, b]=[0,1], \alpha=\beta=1 / 4, p(t)=-t$ for all $t \in \mathbb{R}$ and $h(x, t) \equiv 1$ for all $(x, t) \in[0,1] \times \mathbb{R}$, we have

$$
0=\liminf _{\xi \rightarrow+\infty} \frac{F(\xi)}{\xi^{2}}<\left(\alpha \beta\left(\frac{1}{\alpha+\beta}-\frac{1}{b-a}\right) \frac{k_{2}}{k_{1}}\right) \limsup _{\xi \rightarrow+\infty} \frac{F(\xi)}{\xi^{2}}=\frac{2}{3} .
$$

So, from Theorem 3.3, for each $\lambda>3$, the problem

$$
\left\{\begin{array}{l}
-u^{\prime \prime}+2 u=\lambda f(u) \quad \text { in }(0,1) \\
u(0)=u(1)=0
\end{array}\right.
$$

admits a sequence of classical solutions which is unbounded in $W_{0}^{1,2}([0,1])$.

\section{ACKNOWLEDGEMENTS.}

The authors express their gratitude to the anonymous referees for useful comments and remarks. This research work has been supported by a research grant from the University of Mazandaran.

\section{REFERENCES}

[1] G.A. Afrouzi and A. Hadjian, Infinitely many solutions for a class of Dirichlet quasilinear elliptic systems, J. Math. Anal. Appl. 393 (2012), 265-272.

[2] G. Bonanno and G. D'Aguì, A Neumann boundary value problem for the SturmLiouville equation, Appl. Math. Comput. 208 (2009), 318-327.

[3] G. Bonanno and B. Di Bella, Infinitely many solutions for a fourth-order elastic beam equation, NoDEA Nonlinear Differential Equations Appl. 18 (2011), 357-368.

[4] G. Bonanno and G. Molica Bisci, Infinitely many solutions for a boundary value problem with discontinuous nonlinearities, Bound. Value Probl. 2009, 1-20.

[5] G. Bonanno and G. Molica Bisci, Infinitely many solutions for a Dirichlet problem involving the p-Laplacian, Proc. Royal Soc. Edinburgh Sect. A 140 (2010), 737-752.

[6] G. Bonanno, G. Molica Bisci and D. O'Regan, Infinitely many weak solutions for a class of quasilinear elliptic systems, Math. Comput. Modelling 52 (2010), 152-160.

[7] G. Bonanno, G. Molica Bisci and V. Rădulescu, Infinitely many solutions for a class of nonlinear eigenvalue problem in Orlicz-Sobolev spaces, C. R. Math. Acad. Sci. Paris 349 (2011), 263-268.

[8] G. Bonanno, G. Molica Bisci and V. Rădulescu, Infinitely many solutions for a class of nonlinear elliptic problems on fractals, C. R. Math. Acad. Sci. Paris 350 (2012), 187-191.

[9] G. Bonanno, G. Molica Bisci and V. Rădulescu, Arbitrarily small weak solutions for a nonlinear eigenvalue problem in Orlicz-Sobolev spaces, Monatsh. Math. 165 (2012), 305-318.

[10] G. Bonanno, G. Molica Bisci and V. Rădulescu, Variational analysis for a nonlinear elliptic problem on the Sierpiński gasket, ESAIM Control Optim. Calc. Var. 18 (2012), 941-953.

[11] G. Bonanno and E. Tornatore, Infinitely many solutions for a mixed boundary value problem, Ann. Polon. Math. 99 (2010), 285-293. 
[12] P. Candito, Infinitely many solutions to the Neumann problem for elliptic equations involving the p-Laplacian and with discontinuous nonlinearities, Proc. Edin. Math. Soc. 45 (2002), 397-409.

[13] P. Candito, L. Li and R. Livrea, Infinitely many solutions for a perturbed nonlinear Navier boundary value problem involving the p-biharmonic, Nonlinear Anal. $\mathbf{7 5}$ (2012), 6360-6369.

[14] M. Ghergu and V. Rădulescu, Singular elliptic problems: bifurcation and asymptotic analysis, Oxford University Press, Oxford, 2008.

[15] J. R. Graef, S. Heidarkhani and L. Kong, Infinitely many solutions for systems of multi-point boundary value problems using variational methods, Topol. Methods Nonlinear Anal. 42 (2013), 105-118.

[16] S. Heidarkhani, Infinitely many solutions for systems of $n$ two-point Kirchhoff-type boundary value problems, Ann. Polon. Math. 107 (2013), 133-152.

[17] S. Heidarkhani and J. Henderson, Infinitely many solutions for nonlocal elliptic systems of $\left(p_{1}, \ldots, p_{n}\right)-$ Kirchhoff type, Electron. J. Differential Equations 2012, 69, $1-15$.

[18] S. Heidarkhani and D. Motreanu, Multiplicity results for a two-point boundary value problem, Panamer. Math. J. 19 (2009), 69-78.

[19] A. Kristály, V. Rădulescu and C. Varga, Variational principles in mathematical physics, geometry, and economics: qualitative analysis of nonlinear equations and unilateral problems, Cambridge University Press, Cambridge, 2010.

[20] B. Ricceri, A general variational principle and some of its applications, J. Comput. Appl. Math. 113 (2000), 401-410.

[21] G. Talenti, Some inequalities of Sobolev type on two-dimensional spheres, in: W. Walter (Ed.), General Inequalities 5, Birkhäser, Basel, 1987, 401-408.

[22] E. Zeidler, Nonlinear functional analysis and its applications, vol. II/B and III, Springer-Verlag, New York, 1990.

\section{G. A. Afrouzi}

Department of Mathematics

Faculty of Mathematical Sciences

University of Mazandaran

Babolsar

Iran

E-mail: afrouzi@umz.ac.ir

A. Hadjian

Department of Mathematics

Faculty of Mathematical Sciences

University of Mazandaran

Babolsar

Iran

E-mail: a.hadjian@umz.ac.ir

Received: 4.8.2012. 